\title{
ADJUSTMENT OF GRADUATE STUDENTS WITH ATTENTION DEFICIT HYPERACTIVITY DISORDER (ADHD)
}

\author{
Julien Dalpé, Georgette Goupil, France Landry, \& Rachel Paquette \\ Department of psychology, Faculty of Human Sciences, Université du Québec à Montréal (Canada)
}

\begin{abstract}
The difficulties of undergraduate students with ADHD are widely documented (Emmers, Jansen, Petry, van der Oord, \& Baeyens, 2017). This allows the development of support measures tailored to their needs. Many of these students complete their undergraduate studies and enter master's and doctoral programs. Graduate studies present additional challenges, particularly related to independent research and writing. However, few studies have explored the adjustment of students with ADHD at the graduate level, that is their ability to meet the demands of their study environment. This study aims to compare the adjustment of master's and doctoral students with $\operatorname{ADHD}(n=16)$ and without ADHD. Participants completed a French translation of the Student Adaptation to College Questionnaire (Pariat, 2008, Baker \& Syrik, 1999). This scale measures four dimensions of adjustment: academic, social, personal-emotional and goal commitment/institutional attachment. Students with ADHD present average scores for the overall scale and the four subscales. Moreover, their scores do not differ significantly from those of the students without ADHD. These results indicate that graduate students with ADHD may respond as well to the demands of their study environment as their peers without ADHD, despite the additional challenges associated with their diagnosis. Further studies should explore the conditions and mechanisms facilitating their adaptation.
\end{abstract}

Keywords: ADHD, adjustment, university, graduate students.

\section{Introduction}

The prevalence of Attention deficit and hyperactivity disorder (ADHD) in higher education students is estimated between 3 and $7 \%$ (Emmers, Jansen, Petry, van der Oord, \& Baeyens, 2017). In Quebec (Canada), 6113 university students have requested institutional support regarding a diagnosis of ADHD, which makes ADHD the most frequent mental health diagnosis in Quebec universities (Association québécoise interuniversitaire des conseillers aux étudiants en situation de handicap, 2018). Compared to their peers without ADHD, students with ADHD tend to present lower grade point averages, less developed study skills and greater social and emotional difficulties. They are more likely than their peers to abandon their program or to be on academic probation (DuPaul, Weyandt, O'Dell, \& Varejao, 2009; Weyandt et al., 2013). To help these students to overcome their difficulties, universities offer support services tailored to their needs (Dupaul et al., 2009).

Many students with ADHD manage to complete their undergraduate studies and enter master's and doctoral programs. Graduate studies present new challenges, such as a greater focus on independent research and writing as well as a greater need for adequate time management and work organisation. Despite this, few studies have explored the ability of graduate students with ADHD to meet the demands of their study environment.

According to Baker and Syrik (1984, 1999), adjustment to university has four dimensions. Academic adjustment refers to the ability to meet educational and institutional demands. Social adjustment refers to the integration to the university community as well as the relationships with colleagues. Personal-emotional adjustment is the ability to maintain good physical and mental health despite stress. The last dimension is goal commitment and institutional attachment, that is the feelings toward the institution of attendance and toward university as a whole.

Previous studies found that students with ADHD had lower adjustment than their peers without ADHD (Jones, Rabinovitch, \& Hubbard, 2015; Shaw-Zirt, Popali-Lehane, Chaplin, \& Bergman, 2005). However, no study to our knowledge has investigated the adjustment of students with ADHD at the graduate level in comparison with their peers. This is the aim of the current study. 


\section{Method}

\subsection{Participants}

Participants were 46 French-speaking Canadian master's and doctoral students, including 16 with $\mathrm{ADHD}$ and 28 without ADHD (control group). Table 1 details the descriptive statistics for the participants.

Table 1. Descriptive statistics for the participants.

\begin{tabular}{lcc}
\hline & Students with ADHD $(n=16)$ & Control group $(n=28)$ \\
\hline Gender & & \\
Men & $3(19 \%)$ & $4(14 \%)$ \\
$\quad$ Women & $13(81 \%)$ & $24(86 \%)$ \\
Mean age & 29 years & 28,2 years \\
Educational level & & \\
$\quad$ Master & $7(44 \%)$ & $4(14 \%)$ \\
$\quad$ Doctorate & $9(56 \%)$ & $24(86 \%)$ \\
\hline
\end{tabular}

\subsection{Instrument and procedure}

Participants completed a French translation of the Student Adaptation to College Questionnaire (SACQ; Baker \& Syrik, 1999), either in paper form or online. The SACQ is a 67-item self-reported questionnaire that measures the four dimensions of student adjustment as well as a global adjustment index. Items are answered on a 9-point scale ranging from "does not apply to me at all "to "applies very closely to me ". Scores for the total scale and the four subscales can be converted into $T$ scores $(M=50$, $S D=10)$, a higher score reflecting a higher level of adjustment. The normative sample was composed of 1424 American undergraduate students. For the French translation used in this study (Pariat, 2008), Cronbach's alpha based on the scores of 465 Canadian undergraduate students was .91 for the total scale, .87 for the academic subscale, .85 for the social subscale, .84 for the personal-emotional subscale and .84 for the institutional attachment subscale.

\subsection{Data analysis}

Data were analyzed using the SPSS software, version 25. First, scores for the total scale and each of the four subscales were compiled and converted into $T$ scores. Following the authors' instructions (Baker \& Syrik, 1999), missing data on specific items were replaced with the mean score of the subscale to which the item was associated. Then, the mean score for the total scale and each subscale was calculated for each of the two groups. Scores between 40 and 60 (one standard deviation from the mean) were considered average. Finally, $t$-tests were used to compare the scores of the students with ADHD to those of the control group. A significance level of .05 was used. Effect size was estimated using Cohen's $d$. A $d$ of .20 indicates a small effect, a $d$ of .50 indicates a medium effect and a $d$ of .80 indicates a large effect (Cohen, 1992).

\section{Results}

Table 2 shows the mean scores of the students with ADHD and the control group for the total scale and each of the subscales of the SACQ. Students from the two groups presented average mean scores for the total scale and each of the subscales. There were no significant differences between the two groups. There was a medium, but non-significant difference between the scores of the two groups for the personal-emotional subscale $(d=.49)$, with students from the control group presenting slightly lower scores than those with ADHD.

Table 2. SACQ scores of students with ADHD and control group.

\begin{tabular}{lccccccc}
\hline & \multicolumn{3}{c}{ ADHD } & \multicolumn{2}{c}{ Control } & & \\
\cline { 2 - 5 } & $M$ & $S D$ & $M$ & $S D$ & $t(42)$ & $p$ & Cohen's $d$ \\
\hline Academic & 46.00 & 11.06 & 47.79 & 12.20 & 0.48 & .63 \\
Social & 43.44 & 9.73 & 44.39 & 9.30 & 0.32 & .75 \\
Personal-emotional & 44.06 & 6.85 & 39.64 & 10.05 & -1.56 & .13 & .10 \\
Attachment & 47.63 & 10.36 & 47.29 & 8.46 & -0.12 & .91 \\
Total & 44.38 & 9.68 & 43.96 & 10.58 & -0.13 & .90 \\
\hline
\end{tabular}

\section{Discussion}

The goal of the present study was to measure the adjustment of graduate students with ADHD on the four dimensions defined by Baker and Syrik (1999) and to compare them with a control group of 
graduate students without ADHD. The results suggest that graduate students with ADHD are as well-adjusted as their peers without ADHD. Moreover, students with ADHD may be better adjusted than their peers on the personal-emotional dimension. However, as this difference is not statistically significant, it must be replicated with larger samples. Other studies on the adjustment of students at the undergraduate level showed that ADHD was related to lower adjustment (Jones et al., 2015; Shaw-Zirt et al., 2005). Thus, it is possible that students with ADHD who manage to enter master's and doctoral programs are better adjusted than the average student with ADHD.

As ADHD is the most frequent diagnosis in Quebec universities, the development of support services for graduate students with this condition should be a priority. Further studies should explore the conditions and coping mechanisms that allow these students to respond to the demands of their study environment, including the compensatory strategies developed during undergraduate studies. Studies should also investigate the potential beneficial effects of ADHD in graduate studies, such as creativity.

\section{Contributions, limitations and future directions}

The present study is one of the first to investigate the adaptation of graduate students with ADHD. The results suggest that these students are as well-adjusted as their peers in spite of the additional challenges associated with their diagnosis.

One limitation of the study is that the small size of the sample does not allow to take into account the great diversity of graduate study programs. Some programs require students to conduct a research project of a larger scope than other. The amount of autonomous work required of students can also vary from one program to the other. Thus, it is possible that the adjustment of graduate students, including those with ADHD, is partially dependant on the specific demands of their program. A second limitation is that the number of master's students is greater in the group with ADHD than in the control group. Thus, the two groups are not entirely comparable.

In light of those limitations, future studies should use larger samples that are comparable and representative of the various graduate programs offered in Quebec universities. Various instruments should also be used to investigate the adjustment of graduate students, including objective questionnaires to compare participants and open-ended interviews to better understand their specific experience. Such studies would help to understand the skills, challenges and needs of graduate students with ADHD.

\section{References}

Association québécoise interuniversitaire des conseillers aux étudiants en situation de handicap. (2015). Statistiques concernant les étudiants en situation de handicap dans les universités québécoises, 2014-2015 [Statistics regarding students with disabilities in Quebec universities]. Retrieved from http://aqicesh.ca/docs/AQICESH_stat2017-2018-Sans-univ.pdf

Baker, R. W., \& Syrik, B. (1984). Measuring adjustment to college. Journal of Counselling Psychology, 31, 179-189.

Baker, R. W., \& Syrik, B. (1999). Student Adaptation to College Questionnaire (Manual). Torrance, CA: Western Psychological Services.

Cohen, J. (1992). A power primer. Psychological Bulletin, 112, 155-159.

DuPaul, G. J., Weyandt, L. L., O’Dell, S. M., \& Varejao, M. (2009). College students with ADHD: Current status and future directions. Journal of Attention Disorders, 13, 234-250.

Emmers, E., Jansen, D., Petry, K., van der Oord, S., \& Baeyens, D. (2017). Functioning and participation of students with ADHD in higher education according to the ICF framework. Journal of Further and Higher Education, 41, 435-447.

Jones, H. A., Rabinovitch, A. E., \& Hubbard, R. R. (2015). ADHD symptoms and academic adjustment to college: The role of parenting style. Journal of Attention Disorders, 19, 251-259.

Pariat, L. (2008). Étude des liens entre le soutien social, l'ajustement universitaire et la persévérance en première année de baccalauréat [Study of the links between social support, adaptation to college and perseverance in first year of undergraduate studies] (Doctoral thesis, Université du Québec à Montréal, Montreal, Canada). Retrieved from https://archipel.uqam.ca/2214/1/D1797.pdf

Shaw-Zirt, B., Popali-Lehane, L., Chaplin, W., \& Bergman, A. (2005). Adjustment, social skills, and self-esteem in college students with symptoms of ADHD. Journal of Attention Disorders, 8, 109-120.

Weyandt, L. L., Dupaul, G., Verdi, G., Rossi, J. S., Swentosky, A. J., ... Carson, K. S. (2013). The performance of college students with and without ADHD: Neuropsychological, academic and psychosocial functioning. Journal of Psychopathology and Behavioral Assessment, 35, 421-435. 